\title{
Towards optimization of femtosecond laser pulse nanostructuring of targets for high-intensity laser experiments in vacuum
}

\author{
A. Andreev ${ }^{1,2} \cdot$ J. Imgrunt ${ }^{1} \cdot$ V. Braun ${ }^{1} \cdot$ I. Dittmar $^{3} \cdot$ U. Teubner ${ }^{1,4}$ (I)
}

Received: 1 February 2021 / Accepted: 3 June 2021 / Published online: 28 June 2021

(c) The Author(s) 2021

\begin{abstract}
The interaction of intense femtosecond laser pulses with solid targets is a topic that has attracted a large amount of interest in science and applications. For many of the related experiments a large energy deposition or absorption as well as an efficient coupling to extreme ultraviolet (XUV), X-ray photon generation, and/or high energy particles is important. Here, much progress has been made in laser development and in experimental schemes, etc. However, regarding the improvement of the target itself, namely its geometry and surface, only limited improvements have been reported. The present paper investigates the formation of laser-induced periodic surface structures (LIPSS or ripples) on polished thick copper targets by femtosecond Ti:sapphire laser pulses. In particular, the dependence of the ripple period and ripple height has been investigated for different fluences and as a function of the number of laser shots on the same surface position. The experimental results and the formation of ripple mechanisms on metal surfaces in vacuum by femtosecond laser pulses have been analysed and the parameters of the experimentally observed "gratings" interpreted on base of theoretical models. The results have been specifically related to improve high-intensity femtosecond-laser matter interaction experiments with the goal of an enhanced particle emission (photons and high energy electrons and protons, respectively). In those experiments the presently investigated nanostructures could be generated easily in situ by multiple pre-pulses irradiated prior to a subsequent much more intense main laser pulse.
\end{abstract}

\section{Introduction}

Today the generation of XUV and/or X-ray (both termed $\mathrm{XR}$ ) and/or high energy particle (electrons and protons, termed HEP) emission from the interaction of intense femtosecond laser pulses with solid targets has found wide spread application. Thus various efforts have been made to improve the laser pulse absorption which plays a key role in laser target interaction in general and also the coupling efficiency, related to the emission. Among those efforts, there

U. Teubner

ulrich.teubner@hs-emden-leer.de

1 Institute for Laser and Optics, Hochschule Emden/Leer - University of Applied Sciences, Constantiaplatz 4, 26723 Emden, Germany

2 Saint-Petersburg State University, Universitetskaya Emb. 7-9, St. Petersburg, Russia 199034

3 Physical Chemistry, Hochschule Emden/Leer - University of Applied Sciences, Constantiaplatz 4, 26723 Emden, Germany

4 Carl von Ossietzky University, Carl-von-Ossietzky-Str. 9-11, 26111 Oldenburg, Germany are examples of the strong increase of XR emission by the application of double pulses [1-3], changes of the contrast ratio (main laser pulse to background or uncontrolled prepulses from the amplifier system) [4-6], the optimization of the angle of incidence [7], the target thickness [8] and the target material $[9,10]$, all those performed in the intensity range of $I_{0} \approx 10^{15}$ to $10^{18} \mathrm{~W} / \mathrm{cm}^{2}$, where $I_{0}$ is the laser intensity in the peak of the pulse. In a similar way the yield of HEP emission has also been improved in the same or higherintensity range [11]. More recently, a different approach has been examined for effective proton generation [12] and earlier for X-ray production [13]. Again this makes use of multiple pre-pulses. Here the target surface is structured in situ in a relatively defined way by laser-induced periodic surface structures (LIPSS) $[12,13]$. This is in contrast to the above-mentioned examples where the electron density scale length $[14,15]$ has been optimized to achieve higher absorption, conversion efficiency and emission.

The method of this different approach is rather simple and allows the usage of a high repetition rate target system. Here, first the target surface is nanostructured by the irradiation with tens of pulses with much reduced intensity. Then, second, the main laser pulse at full intensity will arrive and 
"see" an optimal nanostructured surface which significantly enhances the absorption. Thus both the conversion efficiency into fast particles [12] and hard X-ray photons [12, 13] or into soft X-rays and/or XUV emission $[16,17]$ are enhanced as well. In this work, for simplicity of description, we will term the less intense (pre-)pulses which lead to ripple formation as "(ripple) formation pulses" and the full intensity main pulse simply as "main pulse".

As today LIPSS has attracted strong interest in laser micromachining, many investigations have been reported on the subject, see for example $[18,19]$. The origin of the LIPSS formation is largely attributed to the interference of the incident laser wave and the surface scattered electromagnetic wave, thus creating a periodic pattern of the absorbed laser energy along the irradiated surface, as established in [20] for metals and generalized to other materials in [21, 22]. At the beginning of LIPSS formation there is a periodic absorption of laser energy by electrons during the laser pulse. This stage results in a modulation of the lattice temperature along the surface up to tens of picoseconds on metal surfaces [23]. Such a timescale is sufficient for triggering even material ablation [24]. A periodic ablation, which replicates laser energy absorption and material relocation due to recoil pressure of the ablation products, could cause the periodic surface rippling. A longer-term process leads to material removal from the surface layer and plays a role in the final imprinting of the LIPSS on the surface. Such imprinting is performed by laser pulses with relatively low fluence near the ablation threshold [21, 22, 25-28]. This requires tens or even hundreds of laser pulses coupled to the same irradiation spot on the surface to produce the periodic surface relief [29] (see also [30, 31], but note that this work was done with in air environment). Since those early and later works, the possible applications of the rippling production have been studied and developed for scientific and industrial uses.

The generation of laser-induced nanostructures in metals was reported in the context of bulk metals and thin metal films (see, e.g. for $\mathrm{Ti}$ [32-34], $\mathrm{Cu}$ [35], $\mathrm{Al}$ [32], Au [36]). It is known that LIPSS formation depends on the target material [19]. For instance, ref. [35, 37] report that for some metals an increase of laser fluence leads to a decrease of heat transfer by electron-phonon coupling. Then the initial temperature structuring can smooth down by electron diffusion which leads to a weak structuring of the lattice material and finally results in a small relief height [38]. For other metals, such as $\mathrm{Cu}$, this is not the case. Based on the results of Ref. [39] where due to the larger coefficient of electron-phonon (lattice) coupling of $\mathrm{Cu}$, it was found that this metal shows a deeper rippling, our recent work [33] and the present one make use of $\mathrm{Cu}$ targets.

In our recent paper, we have studied the formation of LIPSSs with respect to effective proton generation [33] and first results on ripple formation covering extended areas of thin $\mathrm{Cu}$-foil surfaces are obtained. However, the experiments were performed in air (with the second harmonic of a 120-fs Ti:sapphire laser) and thus chemistry has been expected to play a significant role during the interaction.

The present paper continues the investigation of LIPSS formation on the same metal, but now in vacuum. Presently we have applied a thick polished target irradiated by the first harmonic of a similar laser as before to produce the ripples, now in another parameter range. Moreover, in particular, the dependence of LIPSS, namely the ripple period and height, has been investigated for different fluences and as a function of shot number on the same surface position. Furthermore, to get rid of the influence of ambient gases, and thus more clearly defined interaction conditions, the experiments have been carried out in vacuum. The experimental results and mechanisms for the self-formation of gratings on metal surfaces by femtosecond laser pulses have been carefully analysed and the parameters of the experimentally observed gratings interpreted. Simple scaling formulas for the ripple period and height on the laser parameters have been evaluated. These allow the optimized in situ generation of ripples of an initially flat target by laser pre-pulses with respect to the main laser pulse energy conversion process into fast particles.

The deduction of the optimized generation and the optimized ripple parameters for the aforementioned high-intensity laser interaction experiments has been the goal of the present work. Hence, in contrast to all the previous works, the present one provides very practical parameters which can be directly applied to those experiments on XR production and, in particular, on HEP generation. This includes the information and discussion on the suitable fluence or intensity range, respectively, for the formation pulses and an optimized ripple region size, etc., for superior XR and HEP emission.

\section{Experimental setup}

The experiments were carried out with a chirped pulse amplification Ti:sapphire laser system used for micro- and nanostructuring applications [40]. For the present experiments the system was delivering $\tau_{L}=153 \mathrm{fs}$ (FWHM) linearly polarized pulses at a wavelength of $\lambda_{L}=775 \mathrm{~nm}$ and a maximum pulse energy of $E_{p, \max }=1 \mathrm{~mJ}$. The spatial intensity distribution was Gaussian (slightly elliptical). The pulses were focussed with an achromatic lens of $f=200 \mathrm{~mm}$ focal length onto a flat $\mathrm{Cu}$ sample at normal incidence. Both focussing lens and sample holder were mounted on motorized $x y z$ - and rotation holders, respectively. The setup 
was located in a vacuum chamber evacuated to $10^{-4} \mathrm{mbar}$ $\left(10^{-2} \mathrm{~Pa}\right)$.

The laser light distribution in the focal spot was carefully characterized by a beam profiler measurement. In particular, the image of the focal spot was magnified with a high-resolution microscope objective (numerical aperture 0.4, magnification 20) onto a CCD camera ( $4.4 \mu \mathrm{m}$ pixel pitch). As a result of the measurement we obtained a slightly elliptical-shaped focal spot with the major (long) and minor (short) axis lengths of $d_{m a}=(95 \pm 5) \mu \mathrm{m}$ and $d_{m i}=(75 \pm 5) \mu \mathrm{m}$, respectively $\left(1 / \mathrm{e}^{2}\right.$-width; see Fig. 1 ; average focal spot diameter $\left.d_{L}=85 \mu \mathrm{m}\right)$. In the experiment the applied pulse energy $E_{p}$ was much below $E_{p, \max }$ yielding a maximum possible applied fluence in the peak of $F_{0, \max \approx} 1.5 \mathrm{~J} / \mathrm{cm}^{2}$. Thus the corresponding peak intensity was slightly below $I_{0, \max } \approx 7 \cdot 10^{12} \mathrm{~W} / \mathrm{cm}^{2}$ (averaged over the FWHM pulse duration).

The experiments were performed in vacuum at a laser repetition rate of $1 \mathrm{kHz}$ at 8 different laser pulse energies which lead to 8 different fluences. For comparison, in addition a couple of measurements were performed at atmospheric pressure. In the present experiment the energy was changed by applying different attenuation filters. The focussing scheme and the focal position were always the same. Consequently the measured Gaussian shape in the focal spot distribution remained the same as well and also the laser pulse polarization (note this is important because measurements far off the focal position may add significant p-polarized components in comparison with the s-polarized pulse in focus and this changes the amount of energy deposition).

For each energy a series with different numbers of accumulated shots $N$ were supplied to the same spot on the sample surface. As LIPSS bases on a series of shots (see introduction and related literature), and as a result of our own preliminary experiments, the lowest number of shots $N$ within a series was chosen to be $N=40$. Further series with shot numbers up to $N=120$, and for low fluences even up to $N=200$ were investigated as well. During the sample irradiation the pulse energy was controlled via a calibrated reference detector. To provide clear and well-defined experimental conditions, the sample was shifted to a new position with unaffected surface after each series. To avoid significant ablation, most of the data on ripple formation have been taken at fluences below the damage threshold of the sample $F_{d a m}$ even for the central peak of the pulse. This is because a ring-shaped ripple region (see Fig. 2 below) would be in contradiction to the present goal of target improvement for high laser-intensity interaction experiments where the main pulse hits the same spot as the ripple formation pulses.

The sample of the present experiments was a mechanically polished copper plate (Fig. 1b). Although it has to be mentioned that there are still remaining scratches with depths of a few tens of nanometers and a length between 0.5 and $3 \mu \mathrm{m}$ all over the surface, they can mostly be discriminated during the analysis after the irradiation. This analysis was performed by means an optical microscope (Leica DM4000 B/M), an atomic force microscope (AFM; JPK NanoWizard 3 NanoScience AFM) and a scanning electron microscope (SEM; Zeiss EVO ® MA10).

Most simply the damage threshold was obtained from the images observed with the light microscope and the SEM when ablation is observable in the middle of the spot. More carefully it was determined by a series of independent measurements where the ablation depth and the diameter of the ablation crater, respectively, were measured as a function of fluence (the procedure is described in our previous paper [40] and is based on the usual and well-established simple ablation model; see, e.g. [41]). For the samples of the present work we obtained a value of roughly $0.8 \mathrm{~J} / \mathrm{cm}^{2}$, but one has to note that some incubation effects are present (see Sect. 3).

\section{Experimental results}

As an example Fig. 2 and Fig. 4 show SEM images of the ripple structure obtained with different fluences. More detailed results, in particular, depths and sizes of the
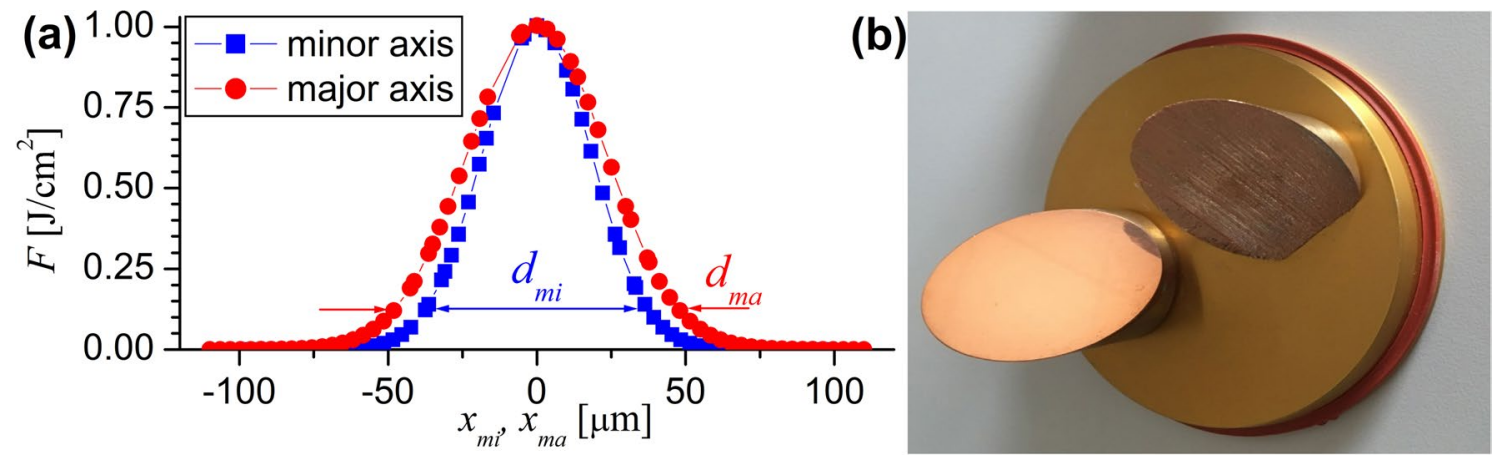

Fig. 1 a Laser beam profile. The $1 / \mathrm{e}^{2}$-width is indicated by arrows. b Target sample used in the experiment 


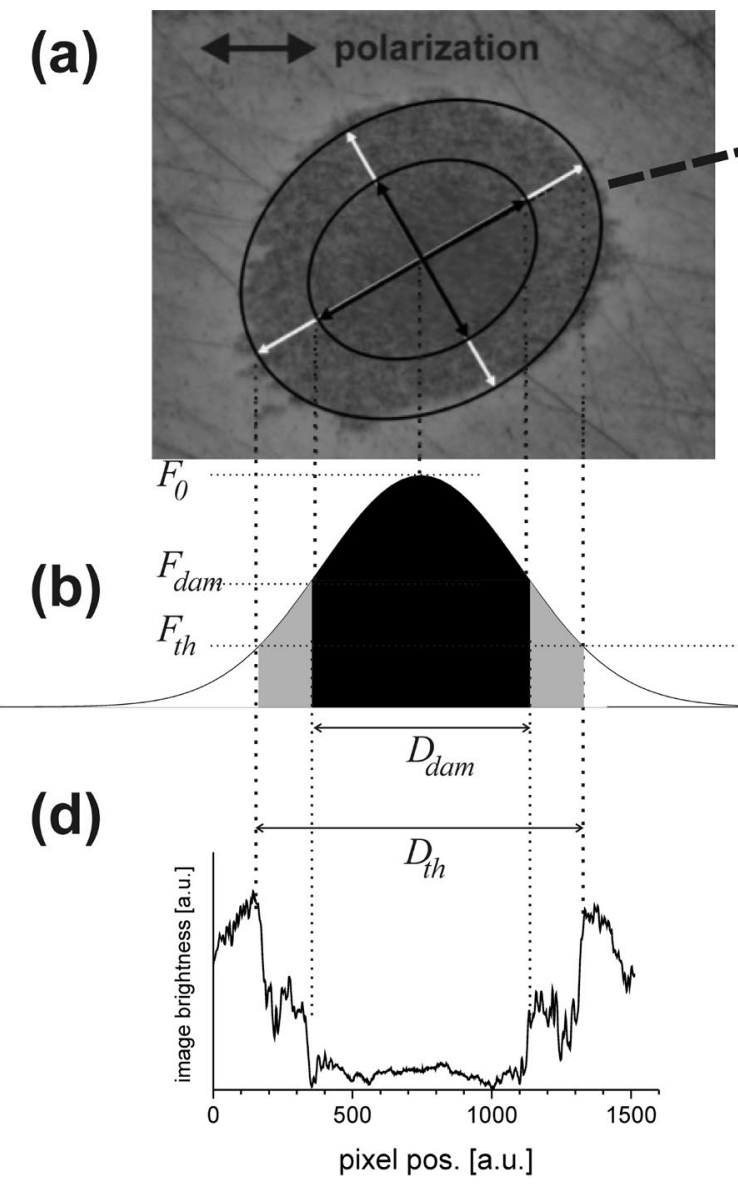

Fig. 2 Illustration of ripple generation on the sample. a SEM image of a sample irradiated with $N=100$ shots at a peak intensity $F_{0}=1.1 \mathrm{~J} / \mathrm{cm}^{2}$. At right an enlarged crop is shown. Further right there is an even more enlarged crop of another SEM image which shows details of the ripple structure $\left(F_{0}=0.6 \mathrm{~J} / \mathrm{cm}^{2}, N=140\right)$. b Illustration of the different regions of surface modification when an intense pulse

ablation-produced cavities, are obtained from the AFM measurements. The deduced ripple parameters then could be related to the irradiation parameters including the laser intensity profiles.

\subsection{Geometry and general results}

Figure 2 illustrates the different experimental situations for measurements above and below damage threshold. It can be recognized that within the laser irradiated region, several zones can be identified. In particular, in the central zone close to the peak fluence $F_{0}$, significant ablation occurs. This is illustrated clearly in Fig. 2b where the Gaussian shape of laser fluence or intensity distribution, respectively, is displayed together with two zones of surface modification. Here the damage threshold of $F_{d a m}$ is exceeded in the vicinity of the laser pulse peak ("damage region"). The threshold region where significant ablation takes place is marked by
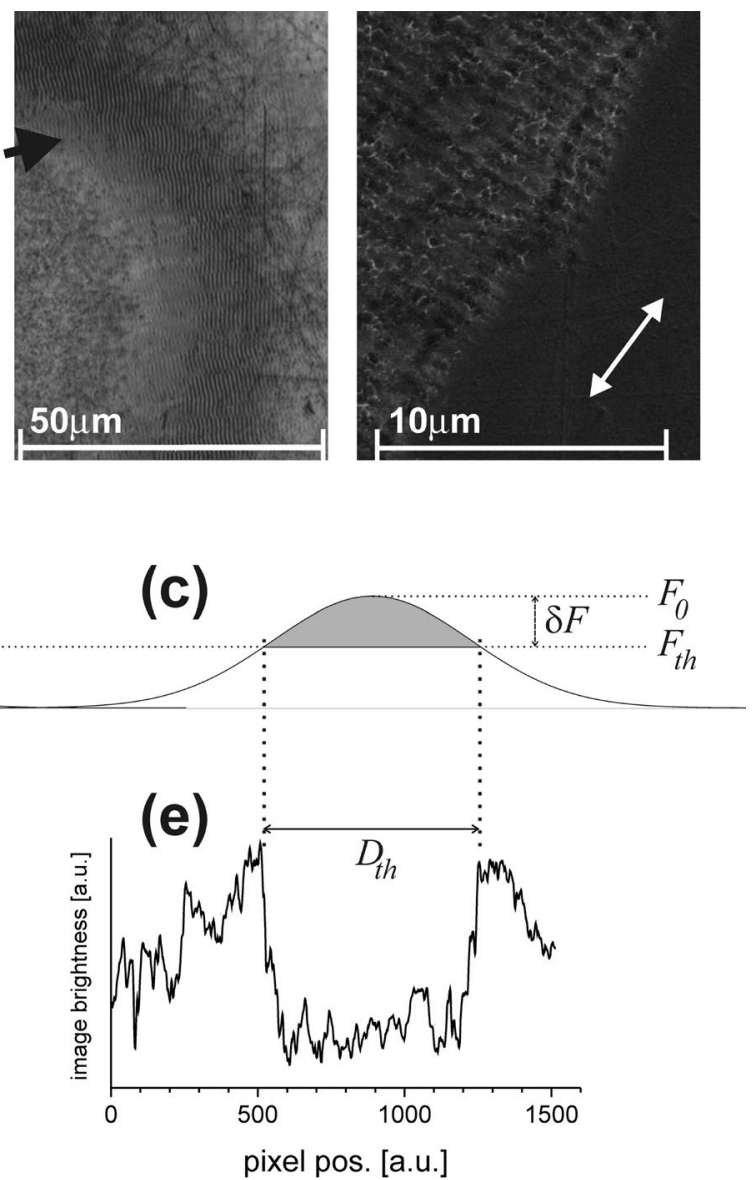

with $F_{0}>F_{d a m}$ irradiates the target: unaffected zone (white), ripple region (grey) and damage region (black). $\mathbf{c}$ same as $\mathbf{b}$, but for a less intense pulse $\left(F_{0}<F_{d a m}\right)$. $\mathbf{d}$ and $\mathbf{e}$ provide the analysis of images captured with a light microscope for the situations of $\mathbf{b}$ and $\mathbf{c}$, respectively (see text)

the inner ellipse (long and short axis lengths of $37 \mu \mathrm{m}$ and $29 \mu \mathrm{m}$, respectively). This is the diameter of the damage crater $D_{d a m}$ and equal to the inner diameter of the ripple region (note that $D_{\text {dam }}$ depends on the direction of the measurement, but this was always properly taken into account). The outer ellipse with long and short axis lengths of $59 \mu \mathrm{m}$ and $47 \mu \mathrm{m}$, respectively, marks the border between the region of ripple formation and the unaffected zone. This is the diameter of the ripple region $D_{t h}$. In the present example the corresponding threshold for ripple formation is $F_{t h}=0.5 \mathrm{~J} / \mathrm{cm}^{2}$ and the damage threshold $F_{\text {dam }} \approx 0.8 \mathrm{~J} / \mathrm{cm}^{2}$.

At lower fluence, ripple formation occurs as long as the fluence is above a certain value $F_{t h}$ ("ripple region"; see Fig. 2c; corresponding SEM and AFM images are displayed in the top right of Fig. 2a and further below). This sets a limit for the onset of such ripple structures. For $F_{0}<F_{t h}$ the surface remains unaffected. 
The SEM images, such as that displayed in the top right of Fig. 2a, show that the boundary between the ripple region and the region where the fluence is below $F_{t h}$ is extremely sharp. This was also observed from the images obtained with the light microscope. The diagrams Fig. $2 \mathrm{~d}$ and e show the brightness within the image along a line through the centre of the ellipse (with some average over neighboured lines above and below; note that those curves do not represent depth profiles!). The brightness curves show that there is an extremely large brightness gradient at the boundary position between the unaffected area and the ripple region. The signal also changes significantly at the boundary position between the ripple and the damage region, respectively. However, here the gradient is much smaller. This leads to less accuracy in damage crater diameter determination from light microscope images. Nevertheless, for the present work even this accuracy is sufficient. We would like to remark that the brightness structures in (d) within the ripple and the damage region, respectively, are not due to ripples because, in contrast to the SEM and AFM images, here the ripples are not resolved. But they result from stray light according to the illumination condition during the microscope analysis.

Figure 2a (and Fig. 4) also shows that the k-vector of the ripples is almost parallel to the laser polarization (indicated by the arrow in Fig. 2a). We write "almost" because due to the slightly astigmatic beam with there are two different foci along the optical axis. But there is a circle of least confusion, which is termed to be the focus in the experiment. However, although the effect is not strong, one has to remark that at this focus position, the polarization is not perfectly linearly s-polarized). This is the result of a symmetry break when compared to a focal spot of a stigmatic Gaussian with perfect s-polarization.

Although a situation as that displayed in Fig. $2 b$ may find applications and although the larger fluence of the pulse in Fig. 2b may have a positive effect on ripple formation when compared to a lower fluence, such a situation with the related ring-shaped ripple profile is not advantageous for the present goal of target optimization by ripple formation pulses. This is because then the main pulse which is aimed to hit the same focal spot, in its central part would hit the damage region. Consequently, this has to be avoided. Thus the situation displayed in Fig. $2 b$ is used for damage measurements, whereas measurements on ripple formation were mostly restricted to peak fluences below (or at least close to) damage threshold (see Fig. 2c).

\subsection{Fluence threshold for ripple formation and damage}

To deduce the threshold limit for ripple formation $F_{t h}$ in dependence of the number of pulses $N$, SEM images were made and analysed. Because the edge is very sharp, the border of ripple formation could be deduced rather easily (see also the light microscope images in Fig. 2d, e). In particular, the corresponding ellipse widths were determined and related to the Gaussian laser beam profile. Then for a given $F_{0}$ and $N$, the threshold could be obtained from the lengths of the major and minor axis lengths, respectively:

$F_{t h}=F_{0} \cdot e^{-\frac{1}{2}\left(\frac{D_{t h} / 2}{d / 4}\right)^{2}}$

where $d$ and $D_{t h}$ are the corresponding values for the major or minor axis, respectively. Multiplication of $F_{t h}(N)$ with $N$ yields the accumulated fluence, i.e. the dose, for the ripple formation threshold. This is shown in Fig. 3.

A fit for all data with the same $F_{0}$ yields a phenomenological relation for the threshold fluence.

$F_{t h}(N)=F_{r 1} \cdot N^{\Gamma_{r}}$

For comparison Fig. 3 shows also the damage threshold data obtained from the present experiment. Here we assumed that damage occurs only for $F_{0}>F_{d a m}$ and we neglected heat propagation effects which is reasonable for fs-laser pulses (see also [42]). For that case the dependence of $F_{\text {dam }}$ on $D_{\text {dam }}$ is equivalent to Eq. (1), and thus, one can deduce the damage threshold from the diameters of the damage crater. But again, we would like to remark that the accuracy in determination of $D_{d a m}$ is lower than that of $D_{t h}$ and consequently the damage data in this figure are less accurate than those for the ripple threshold. This reflects in the symbol size which may be regarded as the size of the error bar.

The negative value of $\Gamma_{r} \approx-0.1$ obtained from Fig. 3 indicates a pulse-induced change of the material or surface property with respect to ripple formation. This relation looks quite similar to the description of accumulation effects by Jee et al. that lead to a damage threshold reduction due to incubation with ns pulses [29] and by Byskov et al. and other groups with fs-pulses [42-44], respectively. These effects usually are described by a relation similar to Eq. (2), i.e. $F_{d a m}(N)=F_{d 1} \cdot N^{\Gamma_{d}}$ where $F_{d 1}$ is the single-pulse damage threshold and $\Gamma_{d}$ a scaling coefficient. But within the experimental error $\Gamma_{r}$ and $\Gamma_{d}$ have notably different values $\left(\Gamma_{d} \approx-0.25\right)$. As material damage and ripple formation result from different physical processes, one cannot expect that the $\Gamma$-coefficients (and the single-shot damage threshold fluences) are the same. But again, as damage measurements are not subject of the present work, we will not consider this further. For ripple formation this will be discussed further in Sect. 4.

\subsection{Ripple period}

Figure 4 and Fig. 5 show examples of an analysis based on a SEM image and an AFM measurement, respectively. 


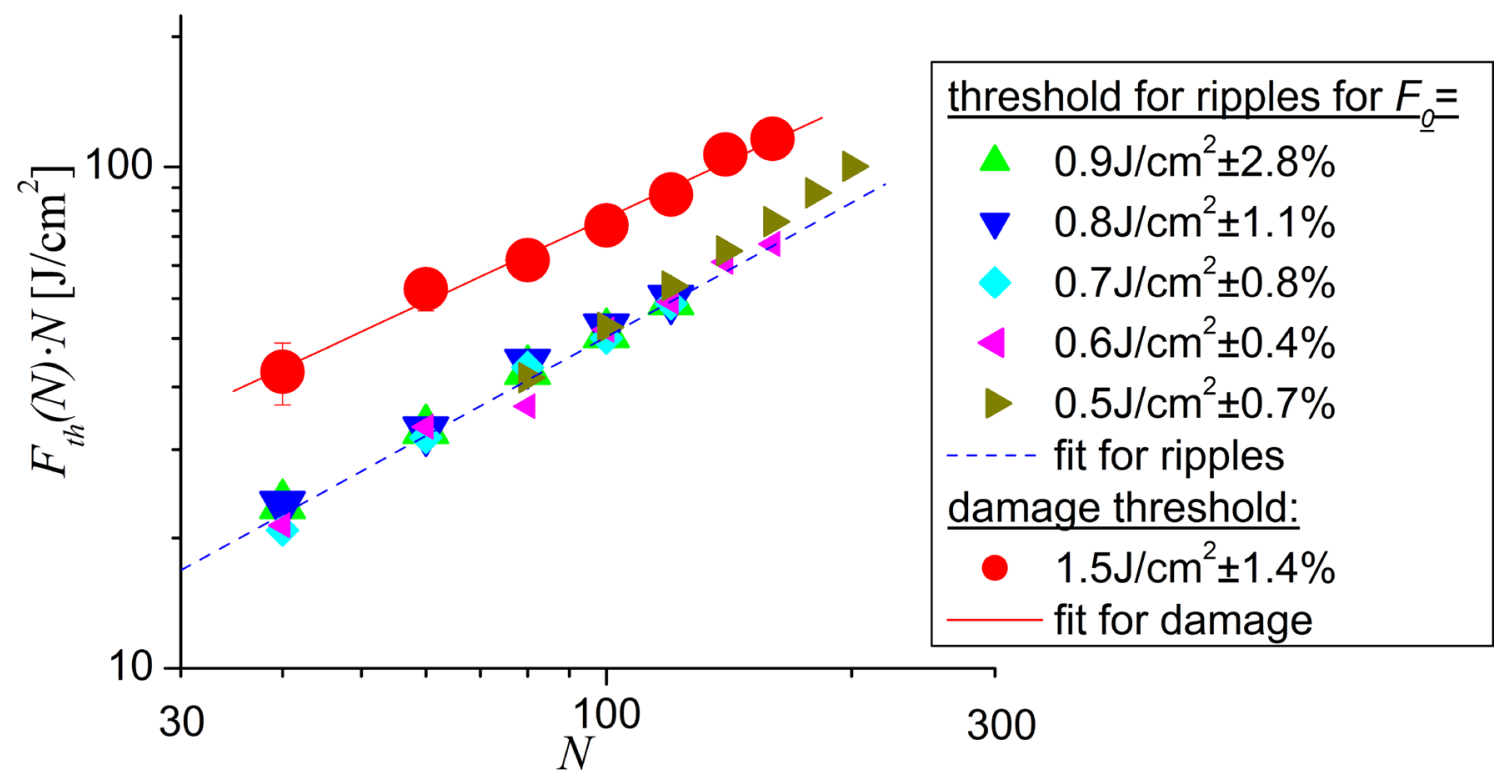

Fig. 3 Dependence of the dose (i.e. accumulated fluence) $F_{t h}(N) \cdot N$, on the number of laser shots $N$. Symbols are experimental data. The dashed line results from a fit to the $F_{0}=0.8 \mathrm{~J} / \mathrm{cm}^{2}$ data (Eq. (1) with
$\Gamma_{r} \approx-0.1$ and $F_{r 1} \approx 0.6 \mathrm{~J} / \mathrm{cm}^{2}$, respectively). For comparison, the red circles represent the values for $F_{d a m} \cdot N$ of the present experiments (a)

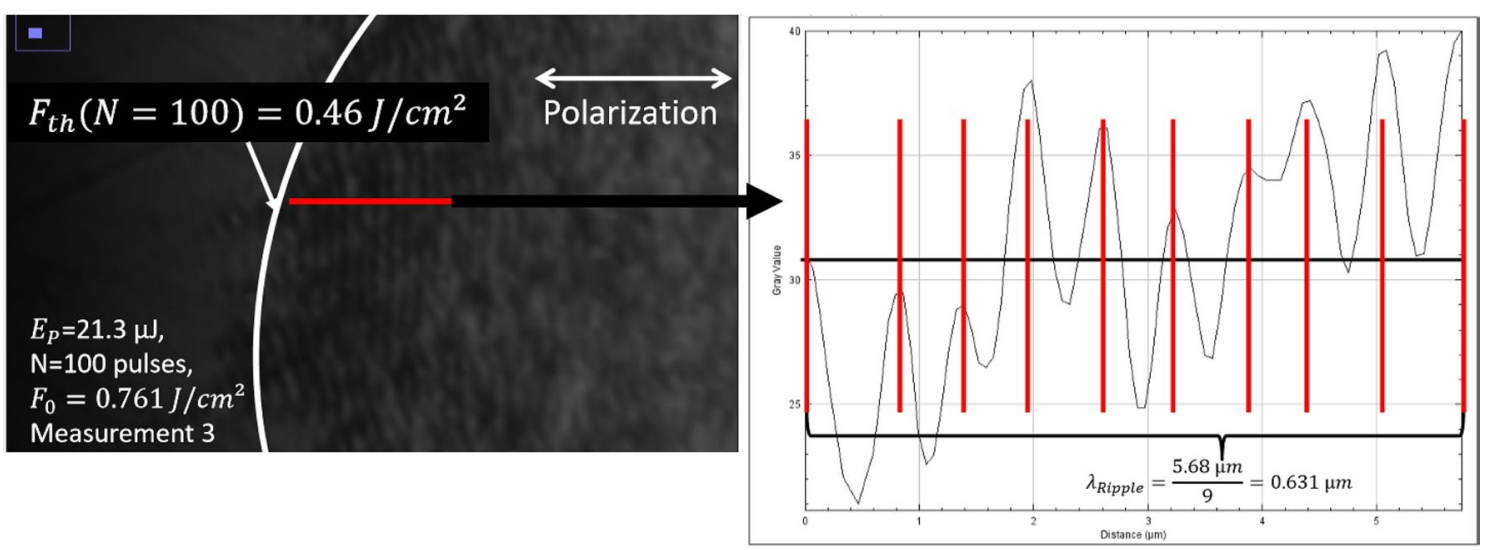

Fig. 4 Analysis based on the SEM image displayed in a. b Ripple profile measured along the red line displayed in a

Figure 4 shows an example of a SEM image-based analysis for $N=100$ shots and $F_{0}=0.8 \mathrm{~J} / \mathrm{cm}^{2}$, i.e. close to $F_{d a m}$ at that shot number. The ripple profile presented in Fig. $4 \mathrm{~b}$ shows the dependence of the modulated surface amplitude in horizontal direction which is almost in parallel to the laser pulse polarization (see white arrow). The white ring in Fig. 4a discriminates the ripple zone from the unaffected zone where the laser fluence is below $F_{t h}$. There may be a small increase in ripple height from the border of ripple formation in direction towards the focal spot centre (see also Fig. 5b). This is attributed to fluence variations within the region where the ripples are produced and, in particular, the increase of fluence towards the spot centre (see Eq. (1) and $\delta F$ in Fig. 2). However, this effect is not very strong.

Another example of an analysis, now for $F_{0}=0.6 \mathrm{~J} / \mathrm{cm}^{2}$, which is significantly below the ablation threshold, and with $N=70$ shots is presented in Fig. 5. Figure 5a shows the AFM "image" and (b) the profile of the ripples, with distances between the maxima (or minima) between 620 and $650 \mathrm{~nm}$. A Fourier analysis of this profile results in a major spatial frequency of 1.54 periods/ $\mu \mathrm{m}$ which corresponds to a period of $649 \mathrm{~nm}$ which is close to the period 

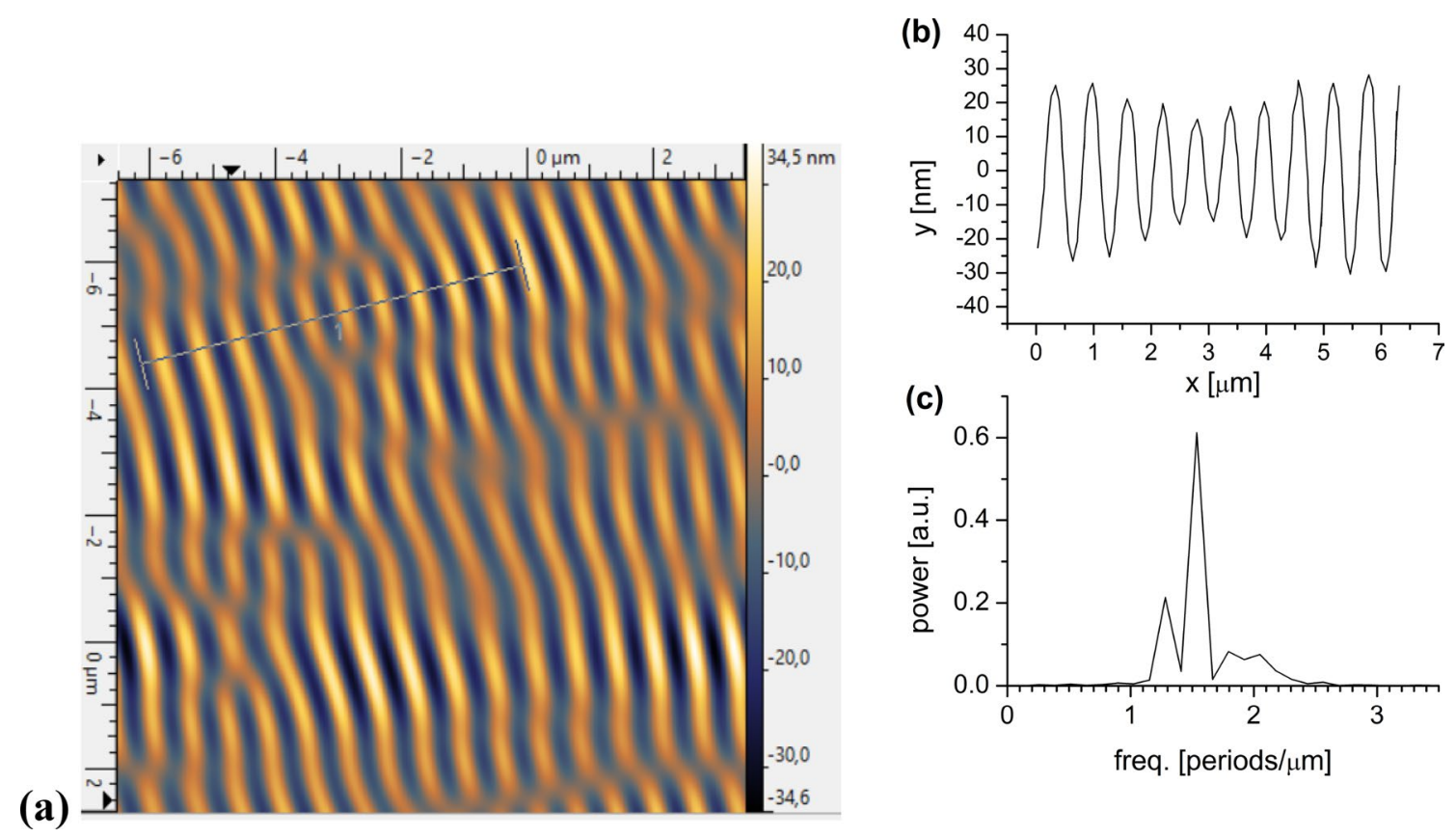

Fig. 5 Analysis based on the AFM image displayed in a: original ripple structure after background subtraction and filtering, displayed in false colours. b Ripple profile measured in the direction perpendicu-

determined from the corresponding SEM measurement. In addition one can observe several neighbouring peaks with corresponding periods from $780 \mathrm{~nm}$ (which is equal to the laser wavelength) down to $390 \mathrm{~nm}$ (Fig. 5c). A significant change of $\lambda_{\mathrm{r}}$ within the ripple region has not been observed (less than error bars).

From the data analysis of Figs. 4 and 5, one can conclude that the average ripple period is approximately $\lambda_{\mathrm{r}} \approx 650 \mathrm{~nm}$. The directionality of the ripples is mainly perpendicular to the laser polarization.

Figure 6 shows the experimental data of the ripple period $\lambda_{\mathrm{r}}$, as a function of the shot number $N$ for 3 different fluences. One can see that for a given $N$, the dependence on $F_{0}$ is weak. This may have been expected because within the ripple region the fluence range is limited between $F_{t h}$ and at most $F_{\text {dam }}$ (see $\delta F$ in Fig. $2 \mathrm{~b}$; for $F_{0}<F_{\text {dam }}$ typically $\delta F<0.5 \mathrm{~J} / \mathrm{cm}^{2}$ ). For this reason also the absorbed fluence $F_{A}$ is rather constant.

In general one may recognize a small decrease in the period. Phenomenologically this may be described by an approximated scaling relation between ripple period and number of pulses as

$\lambda_{r} \approx \lambda_{L} \cdot N^{-0.1}$

We may conclude that altogether, the dependence between the ripple period and the laser fluence is quite week. It can be noted that the decrease of the LIPSS period with pulse lar to the ripple crests. $\mathbf{c}$ Fourier analysis of the profile displayed in $\mathbf{b}$ (power spectrum)

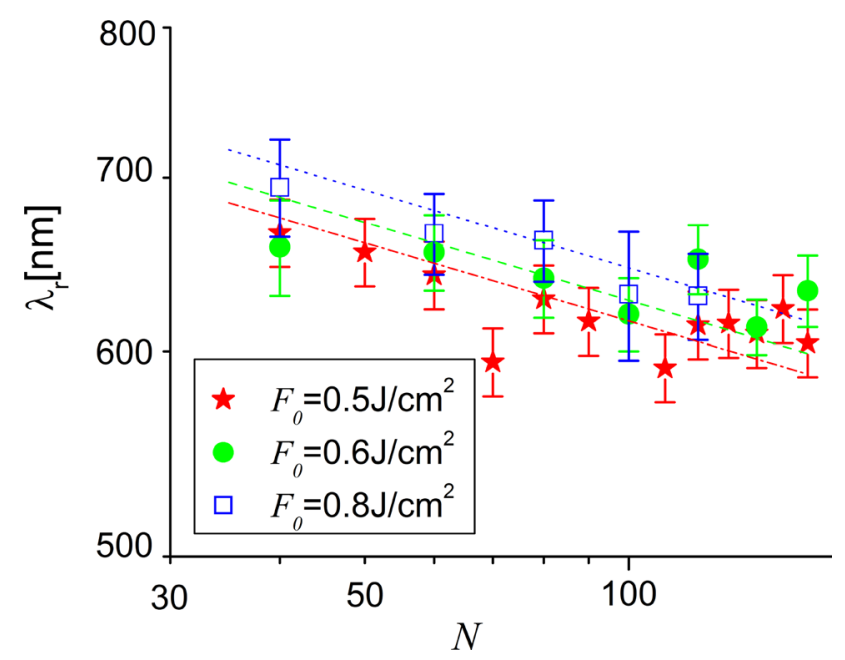

Fig. 6 Dependence of the experimental ripple period $\lambda_{\mathrm{r}}$ on laser shot number $N$ for different laser pulse fluences $F_{0}$ (obtained from the SEM and AFM analysis, respectively). The dotted, dashed and dotted-dashed lines follow scaling relation Eq. (9) in Sect. 4.3 for $F_{0}=0.8,0.6$ and $0.5 \mathrm{~J} / \mathrm{cm}^{2}$, respectively. This is close to the phenomenological scaling of Eq. (3)

number was obtained in [45-48], but for other conditions (usage of other material such as steel or silicon, other angle of incidence, other pulse durations, etc.; see also Sect. 4). Nevertheless, Fig. 6 shows that the ripple period varies a bit with $F_{0}$ an $N$ and allows for some tuning. 
Finally we would like to remark that outside the region indicated by the outer ellipse in Fig. 2 a no ripples were present when ripple generation was performed in vacuum. The surface was always still clean and fully flat. This was in contrast to the additional ripple formation generation experiments performed at atmospheric pressure. At those conditions outside the outer ellipse further ripples were observed, and also frequency components much larger than those corresponding to the periods displayed, e.g. in Fig. 6. However, as noted before, these measurements were taken for comparison only as additional effects induced by air environment are not the subject of the present work. Even so this clearly shows that results of other work on ripple generation outside vacuum cannot be regarded as reliably applicable for the present goal.

\subsection{Ripple height}

Now we would like to discuss the experimental results of the ripple height $h$. First, we would like to remark that, of course, a deduction of $h$ based on the processed AFM images as displayed in Fig. 5 is not useful, but one has to restrict to the AFM raw data. Although this then works well, one has to be aware that there are rather strong fluctuations in height across a selected profile and also for different regions (cf. Figure 4), even if one compares such profiles with same fluence. This makes the deduction of the ripple height quite difficult. Nevertheless, by cross-checking and comparison of various ripple profiles, we may note that although all determined heights result from selected regions of ripples, a careful analysis results in data that seem to be a good representative with the fluctuations taken into account by error bars.

The results are shown in Fig. 7, namely $h$ as a function of $N$. One can clearly observe a significant increase of the ripple height with $N$ until $N \approx 90$. For the lower fluence, then, similar to the observation in [49], there is a slow decrease

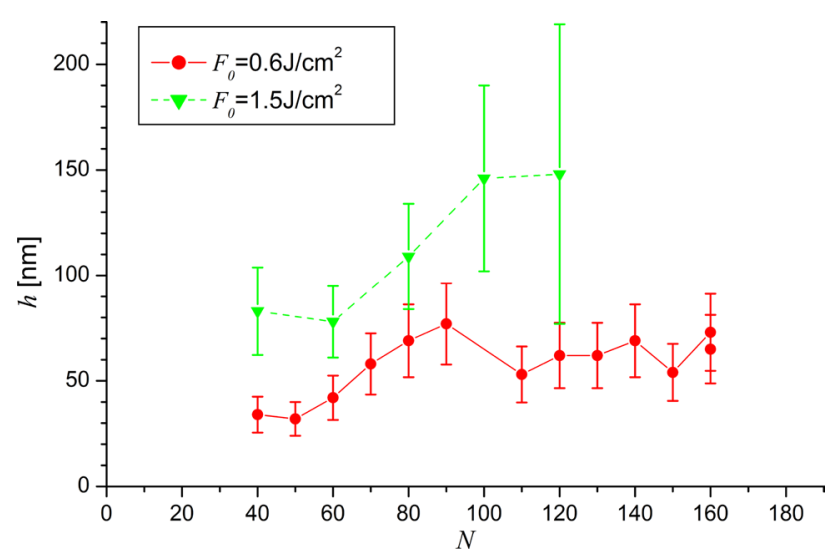

Fig. 7 Ripple height $h$ dependence on shot number $N$ for two different applied fluences (see insert) and finally there is no significant change of $h$ with $N$ within the experimental uncertainty.

A dependence on $F_{0}$ can be recognized by comparison of the results obtained with a low $\left(F_{0} \approx 0.6 \mathrm{~J} / \mathrm{cm}^{2}\right)$ and a high $\left(F_{0} \approx 1.5 \mathrm{~J} / \mathrm{cm}^{2}\right)$ peak fluence, respectively. However, although in the first case the peak fluence is $F_{0} \approx 0.8 \cdot F_{d a m}$, in the second case it is nearly 2 times above the damage threshold (for $N=100$ ). This leads to a damage region in the centre of the rippled area. Even so the larger peak fluence results in a larger ripple height which can be regarded as an upper limit of the ripple heights, again we will not consider ripple generation with $F_{0}>F_{\text {dam }}$ further as this is in contradiction to the goal of the present work (see Sect. 5).

\section{Discussion}

\subsection{Plasma physical effects and simple estimate of the ripple period}

We would like to begin our discussion with a simple estimate of the ripple period, in particular, for the interaction parameters applied within the present experiments: $\lambda_{\mathrm{L}} \approx 775 \mathrm{~nm}$, $\tau_{L} \approx 150 \mathrm{fs}$, solid $\mathrm{Cu}$ target. The fluence on the target $F$ should be sufficiently large for ripple formation, namely $F$ should exceed a threshold necessary for the ripple formation. For the average threshold fluence we assumed a value $F_{\text {th }} \approx 0.5 \mathrm{~J} / \mathrm{cm}^{2}$ (compare to Sect. 3 ). In principle, a ripple estimate could be done by Sipe's theory [, [19, 20] which discusses LIPSS formation on metal weak corrugated surfaces in detail. However, Sipe's theory is mainly valid for a low number of pulses of relatively low fluence [21, 22, 46]. In spite of this, in the present case a relatively high fluence was applied with a peak intensity $I_{0} \approx 10^{12} \mathrm{~W} / \mathrm{cm}^{2}$ which is well above the plasma formation threshold for metal targets. In that range, there is a significant influence of free (generated) electrons and hence plasma physics plays an essential role. That's why surface plasmons become important and a different approach has to be used.

For the present conditions the skin layer of the target can be ionized and a plasma layer can be created. For the present investigation with solid $\mathrm{Cu}$ samples as the target, the skin depth, $l_{\mathrm{s}}=\mathrm{c} / \omega_{\mathrm{p}} \approx 19 \mathrm{~nm}$ ( $c$ is the velocity of light and $\omega_{p}$ $\left(=\sqrt{n_{e} e /\left(\varepsilon_{0} m_{e}\right)}\right)$ the plasma frequency, here taken for the free electron gas of the solid sample). Consequently the energy absorbed by one atom is given by $\varepsilon_{a} \approx F_{\mathrm{th}} /$ $\left(n_{a} l_{\mathrm{s}}\right) \approx 18 \mathrm{eV}\left(n_{a}\right.$ is the atomic number density, with $n_{a} \approx 8 \cdot 10^{22} \mathrm{~cm}^{-3}$ for $\mathrm{Cu}$ ). This is larger than the first ionization potential $U_{i}^{(1)}=7.7 \mathrm{eV}$ and potentially slightly smaller than the second one $U_{i}^{(2)}=20.3 \mathrm{eV}$ [50]. The maximum electron density $n_{e}$ is limited by the average ionization degree $Z \leq 1$, namely $n_{e}=Z \cdot n_{a}$. 
Due to the heating of the plasma bulk to the electron temperature $T_{\mathrm{e}}$, it expands with the ion-sound speed $c_{\mathrm{s}}$. As a result the surface electron density decreases as $n_{e}=n_{e O}\left(l_{s} / c_{s} \tau_{L}\right)$, where $n_{e 0}$ is the electron density directly after ionization. Taking into account some energy losses, one can conclude that the real electron density on the target surface should be $n_{e}<8 \cdot 10^{22} \mathrm{~cm}^{-3}$.

With the refraction index $\mathrm{N}_{\mathrm{r}}$ obtained from Drude's model

$N_{r}^{2}=1-\frac{n_{e}}{n_{c r}}(1+i \beta)$

and the estimates from the model of Bonch-Bruevich et al. [51], one can deduce the grating period at normal incidence as $\lambda_{\mathrm{g}} \approx \lambda_{\mathrm{L}} /\left|\mathrm{N}_{\mathrm{r}}\right|$. Here $\beta=\nu / \omega_{L}$, where $\nu$ is the electron-atom (ion) collisional frequency, $\omega_{L}$ the angular frequency of the incident laser light and $n_{c r}=m_{e} \omega_{L}^{2} \varepsilon_{0} / e^{2}$ the critical plasma density.

For the presently discussed laser pulses the critical (electron) density is $n_{c r} \approx 2 \cdot 10^{21} \mathrm{~cm}^{-3}$ and thus $n_{e} / n_{c r} \approx 40$. This leads to $\lambda_{\mathrm{g}} \approx 0.2 \cdot \lambda_{\mathrm{L}}$ which for the present parameter set $(Z \approx 1, \beta \leq 1)$ yield $\lambda_{\mathrm{g}} \approx 150 \mathrm{~nm}$ which in contradiction to the experimental results (see Sect. 3). On the other hand, this discussion clearly shows that laser plasma physical effects are of significant relevance for such intense pulses as applied within the present work.

\subsection{Advanced estimate of the ripple period}

Thus, we would like to discuss a different way of ripple formation, when compared to the estimate of Sect. 4.1. This is based on the model of Sakabe et al. as the first step [35, 52]. This model describes the modification of the surface of a solid $\mathrm{Cu}$ target by ultrashort laser pulses. Here grating structures are self-formed due to the influence of the laser pulses. The structures are characterized by the following features: the interspaces are shorter than the laser wavelength and they depend on the laser energy density. They become shorter as the laser energy density decreases and the gratings are produced perpendicular to the laser polarization plane.

Following the ideas of that model, the generation of the grating structures is assumed by the following processes. First, via a parametric process (such as in $[53,54]$ ), a femtosecond laser pulse induces a plasma wave on the surface. Then during the surface plasma wave propagation ions become enriched locally. Thus they experience a strong Coulomb repulsion until the peak of the next electron wave arrives at that position. Hence, second, those spatially localized ion clouds Coulomb-explode and expand to vacuum $[35,55]$. Consequently, third, a thin layer is ablated, thus giving rise to the formation of periodic grating structures, which can be regarded as an imprint of a "grating" according to the interspacing of the regions where Coulomb-explosion and thus ablation occurs [56, 57]. If the fluence is large enough (see Sect. 4.1), once such structures are formed by the first pulses of a pulse train, an enhancement process might take place for the subsequent pulses within the pulse train. The electric field is enhanced near the initially imprinted structures, and the strong near field leads to further ablation of the surface, which results in further deepening of the structures [58].

Within this description we further assume that the surface separates the plasma from the vacuum and that one laser pulse (within the pulse train) irradiates the surface at normal incidence where it induces the plasma wave. If we take into account that the laser pulse cannot propagate to a depth beyond the critical density point, then based on the dispersion of the overdense plasma located on the surface, the wavelength $\lambda_{s p}$ of the surface plasma wave can be obtained [51]:

$\frac{\lambda_{s p}}{\lambda_{L}}=\operatorname{Re}\left(\sqrt{\frac{\varepsilon+1}{\varepsilon}}\right)=\sqrt{A+\sqrt{A^{2}+B}} \approx 1-\frac{1+\beta^{2}}{2 \eta}$

where the dielectric function is given by

$\varepsilon=1-\frac{\eta}{1+\beta^{2}}+i \beta \frac{\eta}{1+\beta^{2}}$

the relative density $\eta=n_{e} / n_{c r}>1$ and

$A=\frac{\varepsilon_{\mathrm{r}}\left(1+\varepsilon_{\mathrm{r}}\right)+\varepsilon_{\mathrm{i}}^{2}}{2 \cdot\left(1+\varepsilon_{\mathrm{r}}\right)^{2}+\varepsilon_{\mathrm{i}}^{2}}$

$B=\frac{\varepsilon_{\mathrm{i}}^{2}}{4 \cdot\left(1+\varepsilon_{\mathrm{r}}\right)^{2}+\varepsilon_{\mathrm{i}}^{2}}$

$\varepsilon_{r}$ and $\varepsilon_{i}$ are the real and imaginary part of $\varepsilon$, respectively. Taking into account that according to the expansion within the skin depth of the overdense surface plasma, $\eta \approx 10$ and $\beta \sim 1$, then from Eq. (5) one obtains $\lambda_{s p} \approx 0.85 \lambda_{L} \approx 650 \mathrm{~nm}$ which is the period of the imprinted grating. As a result, it is concluded that the ripple period corresponds to the wavelengths of the induced plasma wave, i.e. $\lambda_{g}=\lambda_{s p}$. This is in agreement with the experimental results, which indicates that for a laser fluence of approximately $0.5 \mathrm{~J} / \mathrm{cm}^{2}$, the average ripple period $\lambda_{r}=\lambda_{g}$ is approximately $\lambda_{r}=650 \mathrm{~nm}$ (see Fig. 3).

It is important to note that the orientation of the observed grating structure discussed here is perpendicular to the laser polarization plane. It is reasonable to conclude on the basis of this fact that the plasma waves are driven by the electric field of the light pulse. We would like also to remark that by changing the angle of incidence one can significantly increase the ripple period (see for example [51, 59]) and by decreasing of the laser wavelength (using, e.g. harmonics) 
one can significantly decrease this period. Further influence on the ripple period may be taken by the number of shots $N$ within the pulse train and their fluence. This will be discussed in the next section.

\subsection{Dependence of the ripple period on the number of shots within the pulse train and on the applied laser fluence}

From the discussion in the previous section it becomes clear that when the relative plasma density $\eta$ changes, the structure period will change as well. To discuss this, we consider the electron density $n_{\mathrm{e}}$ of the plasma bulk in the skin layer produced by the laser on the surface. As we will see below, the average ionization degree follows a power relation on the laser energy fluence and thus $n_{e}=Z n_{a} \propto F^{\zeta}$, where $\zeta<1 . T_{\mathrm{e}}$ follows also a power law, namely $T_{\mathrm{e}} \propto F^{\xi}$ ( $\zeta$ an $\xi$ are scaling coefficients).

To estimate the scaling of the electron temperature $T_{e}$ and the average ionization degree $Z$ on the intensity, similar to [7], we have applied a self-consistent calculation using a delta-pulse model for laser pulse absorption [60] together with a high-temperature Fermi model [61] and nonlinear heat conduction [62]. From this we find $\zeta \sim 0.1$ and $\xi \sim 0.5$ (in the intensity range of relevance for the present experiment, namely approximately $I_{0} \sim F_{0} / \tau_{L} \sim 10^{12} \mathrm{~W} / \mathrm{cm}^{2}$; this is consistent with the experimental data in [63]). Therefore, the normalized surface electron density is related to the laser energy density as $\eta \propto n_{\mathrm{e}} \propto Z \propto F^{0.1}$ and the relative collisional frequency $\beta \propto n_{\mathrm{e}}{ }^{\sigma} T_{\mathrm{e}}^{\mu}$ (where for $T_{\mathrm{e}}<1 \mathrm{eV}: \sigma=0, \mu=2$ and for $T_{e} \geq 5 \mathrm{eV}: \sigma=2, \mu=-3 / 2$ ) [58]). Applying these relations together with Eq. (5) for the present conditions, the spatial modulation may be approximated as follows:

$\lambda_{r} \approx 1.15 \cdot \lambda_{L} \cdot\left(\frac{F_{0}}{F_{A} N}\right)^{0.1}$

where $F_{A} \cdot N$ is the absorbed dose (i.e. the absorbed accumulated fluence).

For the discussion on the laser fluence (or intensity), we have to note that the applicable fluence range for ripple formation is rather limited (see Fig. 2). According to the experimental data or according to the scaling relations (see Eq. (2) and the similar relation for $F_{d a m}$ together with the experimentally determined scaling parameters), one obtains a feasible fluence range of $F_{0} \approx 0.4$ to $1 \mathrm{~J} / \mathrm{cm}^{2}$ for $N=40$ and $F_{0} \approx 0.3$ to $0.7 \mathrm{~J} / \mathrm{cm}^{2}$ for $N=160$. Within such a small fluence range one cannot expect a significantly different interaction with the surface plasma. Consequently for the applied range of peak fluences the absorbed fluence is approximately constant $\left(F_{A} \approx 0.2 \mathrm{~J} / \mathrm{cm}^{2}\right)$ and a weak dependence on the incident peak fluence and the number of applied shots is present by the weak scaling on $F_{0}$ and $N$, respectively.
Of course, this relation can only be obtained numerically. It results in an interpolation of the exact dependence $\lambda_{S P} / \lambda_{\mathrm{L}}(F, N)$, which follows from Eq. (5).

Equation (9) agrees with the experimental results (see Eq. (3) and Fig. 6). But we may note that the present interpretation cannot be applied to laser pulses with a fluence below $0.2 \mathrm{~J} / \mathrm{cm}^{2}$ since below this threshold there is only little plasma on the metal surface. This sets the lower limit which also is in good agreement with the ripple threshold limit experimentally observed in Sect. 3.2.

\subsection{Estimate of the ripple height}

In a second step we have to take into account the relaxation processes. Destabilization of the lattice on a timescale of several hundreds of femtoseconds has been observed in [64]. Accordingly, for the conditions of the present experiments the target surface is expected to be modified on some depth.

If one supposes that a significant part of the laser energy is transferred into plasmons with a corresponding wavelength $\lambda_{S P}$ by forming optimal grating parameters, then from the method described in ref. [65] one can estimate a grating depth $h$ of the order of $50 \mathrm{~nm}$. On the other hand, even in case of less optimized plasmon generation, a periodic electron temperature profile with the same period transfers to the lattice profile and leads to a periodic heating and then a modification (melting) of the surface. In that case one can suppose that the $i$-th laser pulse produces first a heated and then a melted target volume that is approximately given by $V=h_{i} \cdot \lambda_{s p} \cdot d_{L}$.

Here the melted area is given by $\lambda_{s p} \cdot d_{L}$ and the melting depth by $h_{i}$. When $F$ is the incident laser pulse fluence, then from energy conservation one can estimate the absorbed fluence $F_{A}=A_{s i} F \cdot \lambda_{\mathrm{sp}} \cdot d_{L}$ from the material density $\rho$, the surface absorption coefficient $A_{s i}(\leq 1)$ and the supplied heat $Q=c_{h} T_{\text {melt }}+Q_{\text {melt }}($ in $\mathrm{J} / \mathrm{g})$ :

$F_{A}=\rho \cdot h_{i} \cdot \lambda_{s p} \cdot d_{L} \cdot Q+F_{r} \cdot\left\{\lambda_{s p} \cdot d_{L}+2 h_{i} \cdot\left(\lambda_{s p}+d_{L}\right)\right\}$

Here $F_{l} \approx A_{l i} F$ describes fluence losses by different processes. $A_{l i}$ is the related loss coefficient, $c_{h}$ the heat capacity, $T_{\text {melt }}$ the melting temperature and $Q_{\text {melt }}$ the specific melting heat, with $Q_{\text {melt }}=213 \mathrm{~J} / \mathrm{g}$ for $\mathrm{Cu}$.

Consequently, one can estimate the ripple height generated by the $i$-th laser pulse within the pulse train

$h_{i} \approx \frac{A_{s i} \cdot F \cdot-F_{l}}{\rho \cdot Q \cdot \lambda_{s p} \cdot d_{L}+2 F_{l} \cdot\left(\lambda_{s p}+d_{L}\right)} \cdot \lambda_{s p} \cdot d_{L}$

It is important to note that although melting is only an issue for timescales much longer than the pulse duration, here it does play some role because there is enough time for melting and evaporation after the first pulse before the 
second one arrives and so on. Therefore, for the present conditions the estimated ripple height after irradiation with $N$ pulses and summation of its influence, according to (11), may be estimated as follows:

$h=\sum_{i=1}^{N} h_{i}=\sum_{i=1}^{N} \tilde{A}_{s i} h_{0} \approx \tilde{A}(N) \cdot \frac{F}{\rho} Q$

where $\tilde{A}_{s i}=A_{s i}-A_{l i}$,

$h_{0}=F \cdot\left(\rho \cdot Q \cdot\left(1+2 F_{l} \cdot \frac{\left(\lambda_{s p}+d_{L}\right)}{\rho \cdot Q \cdot \lambda_{s p} \cdot d_{L}}\right)\right)^{-1} \approx \frac{F}{\rho \cdot Q}$

and

$\tilde{A}(N)=\sum_{i=1}^{N} \tilde{A}_{s i} \approx 0.8 \frac{\sqrt{N}}{\sqrt{N+10}}$

The latter approximation follows from comparison with the experimental data.

With these estimates and the present set of parameters one can estimate $h \approx 50 \mathrm{~nm}$ which is close to the experimental data shown in Fig. 7. In particular, the dependence on $N$ of the experimental results may be reproduced by Eq. (12) and Eq. (14). Starting from the initial surface condition, according to Eq. (12), $h$ is increased for the following pulse and thus the absorption is enhanced [66,74-76]. But as the ripple becomes much deeper the additional influence on absorption becomes poor and saturation is reached which results in an approximately constant value of $h$ independent of $N$. This saturation onset then may be regarded as the optimized number $N_{\text {opt }}$ of weak pre-pulses for surface conditioning prior to the strong main pulse in the high-intensity laser interaction experiments. For the present conditions: $N_{\text {opt }} \approx 90$ to 100 .

\section{Applicability of nanostructured targets}

In the following we would like to discuss the applicability of (in situ) nanostructured targets for an efficient generation of XR and/or HEP by an intense main pulse subsequent to the irradiation with multiple less intense ripple formation pulses.

Figure 2 and Fig. 8 indicate that low (near-laser wavelength) spatial frequency ripples (LSFR) are quite distinct and well defined (see Fig. 6). From Fig. 7 one recognizes that the depth of such a structure on a metal surface can be approximately $50 \mathrm{~nm}$ to $>100 \mathrm{~nm}$. The obtained periods and depths are essential for laser HEP generation and acceleration [34] and XR generation. Thus LSFRs can be considered as good candidates for these applications.

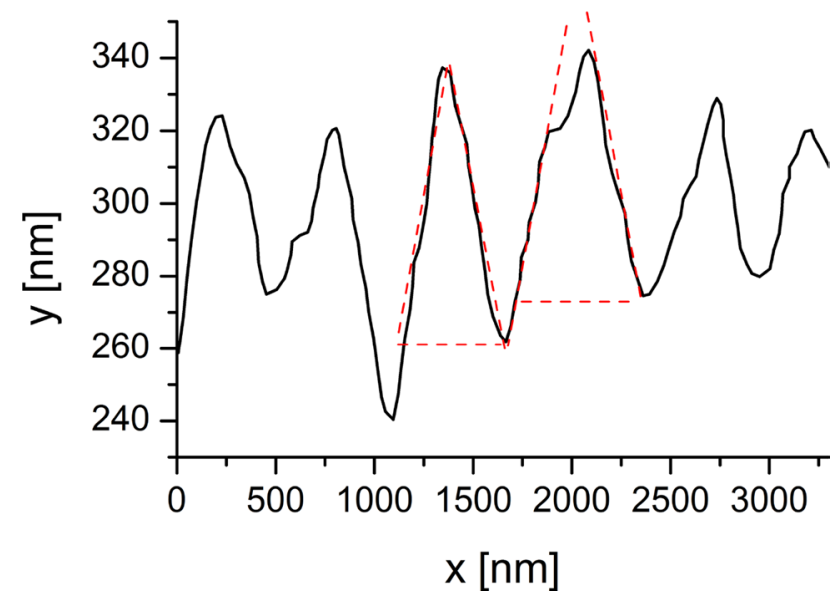

Fig. 8 Typical ripple profile obtained from an experiment with $F_{0} \approx 0.6 \mathrm{~J} / \mathrm{cm}^{2}, N=80$ (determined from the unprocessed AFM raw data image). The dashed lines indicate an approximated triangle profile (see text)

To evaluate the applicability of laser-induced nanostructures suitable for laser particle acceleration, etc., a theoretical analysis was performed for flat and structured $\mathrm{Cu}$ targets, respectively. From Fig. 8 one can approximate the shape of the modulation (ripple) by a triangle with a base between 300 and $350 \mathrm{~nm}$ and a height of 50 to $70 \mathrm{~nm}$. This is used as the input for the simulations, together with the laser pulse parameters of the ripple formation pulses and those of the main pulse.

The simulation results show that a flat foil is an inefficient converter for high energy particle generation, whereas if additionally a surface distortion is considered, the situation changes strongly (typically thin flat foils are used as targets in proton acceleration experiments). The simulations demonstrate that a significant enhancement of absorption up to $100 \%$ (60\% in [67]) with an appropriate surface structure is possible. In [68] for nearly optimal parameters, it was shown that the advantage of the rippling seems to be more connected with the number of accelerated ions than with their maximal energy, which is of interest, e.g. in dose deposition.

A general remark on laser particle acceleration and $\mathrm{X}$-ray generation in the presence of a ripple profile is that the absorption is strongly increased on the relief crest and is slightly decreased besides it $[68,69]$. The optimum relief height decreases with increasing main pulse laser intensity (see also [67]). If well adapted, nanostructuring increases the energy transfer from the laser pulse to the electrons. Also proton acceleration could be significantly improved in terms of maximum energy and proton number [12]. Thus, by using the optimization formulas for proton acceleration from [12] and our eqs. (3), (5), (9) and (11), one can get the optimal main laser pulse parameters (intensity and duration approximately $10^{19} \mathrm{~W} / \mathrm{cm}^{2}$ and $30 \mathrm{fs,}$ 
respectively) and also the optimal ripple formation pulses (of the order of 100 pulses with an intensity of $10^{12} \mathrm{~W} / \mathrm{cm}^{2}$ and a FWHM duration of $150 \mathrm{fs}$ ) for an efficient acceleration and generation processes. Here we would like to note that although the ripple period and its height are essential for the profile optimization (as discussed within the present work), the specific profile asperity (rectangular, triangular or harmonic) is of minor importance because this only has a slight influence on the absorption [70] and the X-ray yield [71]. Some tuning of $\lambda_{\mathrm{r}}$ may be obtained by adapting $N$ and/or by changing the angle of incidence and/or by changing the wavelength of the ripple formation pulses.

Altogether, the optimal relief increases the absorption to a maximum value and improves the XR photon yield. It may also influence the absorption process itself and, e.g. lead to an increase of the collisionless interaction of the main pulse with the target. This may be important because the improvement of the XR yield does not only depend on the amount of the absorption itself, but also on the mechanism of how the laser pulse is absorbed [63]. The change of the relief parameters allows one to selectively influence the energy distribution function of the hot and cold electron ensembles and thus to achieve the maximum energy of the protons or the maximum $\mathrm{K}-\alpha$ yield [71]. According to our modelling for the present parameters, the K- $\alpha$ yield can be increased by optimal rippling by approximately a factor 3 and the hot electron number by a factor of 5 .

However, we would like to comment that optimization is not the same for protons as it is for $\mathrm{K}-\alpha$ emission. If the target parameters are chosen such that the laser absorption is maximized, the temperatures and numbers of the hot electrons may be maximized and accordingly the numbers and energy of the protons as well. But for those conditions the cold electron temperature may not correspond to the maximum K-shell ionization cross section [72]. In such a case, the $\mathrm{K}-\alpha$ yield will not be maximum despite the subsequent cooling of the hot electrons and their transformation into cold electrons [73]. Then, for a fixed laser intensity, the optimum relief parameters do not coincide in order to achieve the maximum proton energy and the maximum $\mathrm{K}-\alpha$ yield at the same time [12]. This means that the main pulse parameters have to be adapted to the intended optimized interaction.

Besides these very basic physical issues, there are practical issues for optimized XR and HEP sources as well. In particular, for HEP generation an important issue is the shape and the size of the region where ripples are generated. For the experiments with the high-intensity main pulse it is preferable that the ripple formation pulses generate a rather homogeneous region with ripples and this with the additional demand that there is no damage in its centre. Furthermore, this region should cover a significant fraction within the FWHM diameter which is the by far most important region for the nonlinear interaction of the main pulse with the target during XR or HEP generation, respectively.

We will discuss this for Gaussian-shaped pulses as those are typical for high-intensity laser plasma experiments. Thus, according to Fig. 2, the applied peak fluence has to be chosen to be close to, but below the damage threshold. Thus a ring-shaped ripple region is avoided which would be in contradiction to the requirement. In that case one obtains a disc-shaped ripple region with rather homogenous ripples (remember from end of Sect. 4.3 that the fluence range within the ripple region is rather limited). Moreover, for $N \approx N_{\text {opt }} \approx 100$ the diameter of the rippled area comes close to the FWHM of the laser spot which well fulfils also that requirement. This is shown in Fig. 9.

We are aware that the previous discussions are somewhat sophisticated. For this reason, the main results are summarized in Table 1.

Finally, we would like to remark that the presently proposed structuring method is easy to implement for any laserdriven secondary source of high energy particles (photons, electrons, protons), is robust, in situ, and can be applied in between high power laser shots at a high repetition rate. For example, within a complex laser system, the low-intensity pulses can be generated by the $\mathrm{kHz}$ repetition laser preamplifiers and the high-intensity main pulses by, e.g. a $10-\mathrm{Hz}$ main amplifier.

Although there is still some potential progress for the optimization of the interaction conditions, the present work contributes to a significant progress with respect to laserdriven emission source efficiency.

\section{Summary}

In summary, we have investigated the generation of laserinduced periodic surface structures (LIPSS) on flat solid $\mathrm{Cu}$ targets with respect to their optimization when used as targets for interaction experiments with high-intensity laser pulses. In particular, this includes their usage as femtosecond-laser-driven secondary sources of high energy particles (photons, electrons, protons) to obtain an improved efficiency.

The present experiments have been carried out with approximately 150 fs pulses (FWHM) with a Gaussian radial intensity distribution with a maximum peak intensity below $10^{13} \mathrm{~W} / \mathrm{cm}^{2}$. These are the ripple formation pulses which correspond to potential pre-pulses in the interaction experiments performed at much higher intensities with the subsequent main pulses.

The periodic surface structures have been carefully analysed by means of an optical microscope, a SEM and an AFM. In particular, experimental data for the ripple period and height have been obtained as a function of the 


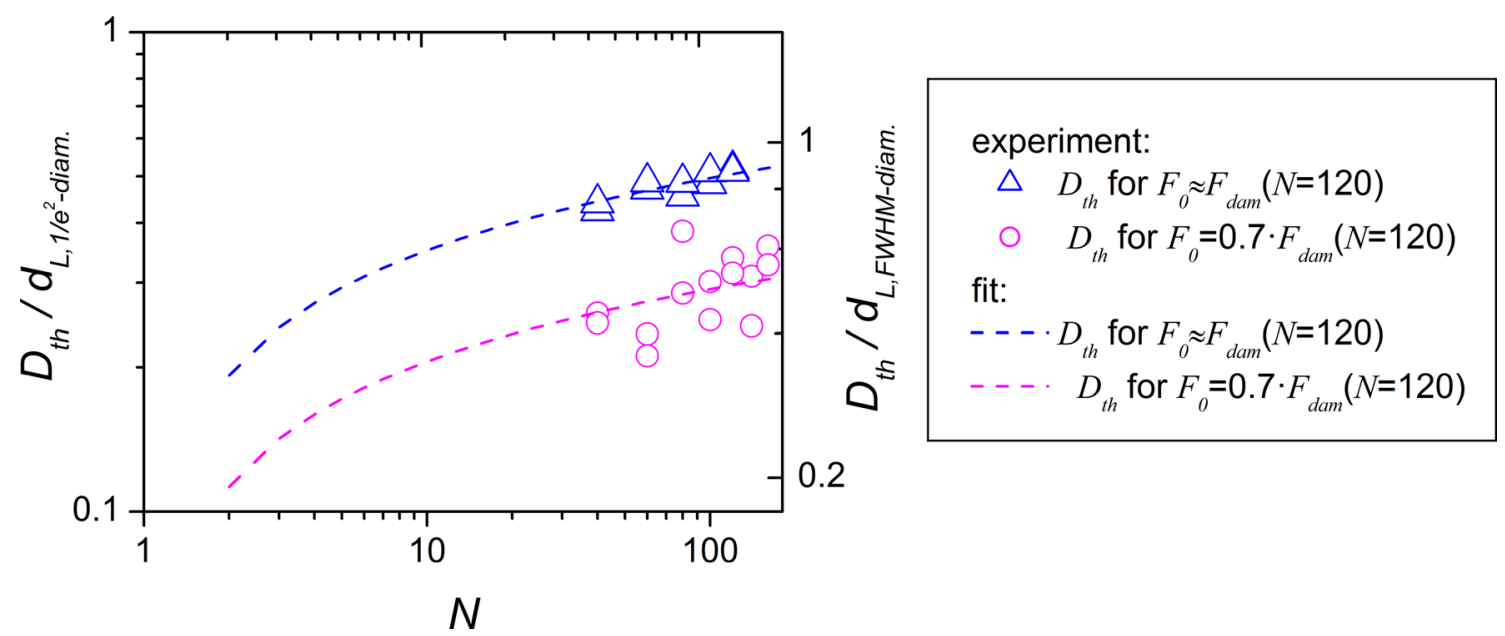

Fig. 9 Experimentally determined diameter of the region where the threshold for ripple formation is exceeded, but below $F_{d a m}$. The dashed lines are the fits according to Eq. (2). The extrapolation to low values of $N$ might not be correct. It may just indicate how the diame-

laser fluence and the number of applied pulses. The threshold for ripple formation shows a weak dependence on the pulse number and has a value between 0.4 and $0.5 \mathrm{~J} / \mathrm{cm}^{2}$ (for a peak fluence between 0.5 and $0.8 \mathrm{~J} / \mathrm{cm}^{2}$ and shot numbers between 40 and 200 shots). It can be described by a simple empirical scaling law. The threshold for material damage is $F_{\text {dam }} \approx 0.8 \mathrm{~J} / \mathrm{cm}^{2}$ (for $N \approx 100$ ).

The LIPSS process involving the interaction of femtosecond laser pulses with surface plasma waves, as well as the excitation of a surface solid-state plasma, has been discussed. The relation of the experimentally observed ripple parameters on the laser fluence and pulse number is well described by analytical scaling relations. The shapes, heights and distances can be controlled by choosing the right number of incident pulses. The peak laser fluence ter of the area of ripple formation increases during formation process which begins not too far from the damage fluence at the laser peak (in that sense an experimental verification is not necessary; this is due to the reduction of $F_{t h}$ with $N$ according to Eq. (2))

plays a minor role but should be below damage threshold. The processing method can be easily applied to relatively large areas and is shown to create a sufficiently homogeneous structured surface.

It has been demonstrated that laser-induced nanostructures can be produced in a parameter range which matches well for applications such as the enhanced generation of $\mathrm{XR}$ and/or HEP emission (increase by a factor 3 to 7). In order to evaluate this approach in more detail, resourceconsumptive numerical simulations in comparison with experimental studies are potential next steps. However, although this is a subject of future work, the present estimates and scaling laws are already sufficient for the application of in situ optimized targets. 
Table 1 Important parameters for improved XR and HEP generation and achieved results. Note: there are further issues that cannot be easily tabulated (see text). The parameters of the main pulse (not a subject of the present work) are marked with grey background. As usual for high-intensity laser interaction experiments, Gaussian pulse shapes are assumed for both, main pulse and ripple formation (pre-) pulses

\begin{tabular}{|c|c|c|c|}
\hline & $\begin{array}{l}\text { general requirement } \\
\text { and demands from } \\
\text { theory for efficient XR } \\
\text { generation }\end{array}$ & $\begin{array}{l}\text { general demands and } \\
\text { demands from theory } \\
\text { for efficient HEP } \\
\text { generation }\end{array}$ & $\begin{array}{l}\text { results of the from } \\
\text { present work }\end{array}$ \\
\hline $\begin{array}{l}\lambda_{\mathrm{L}} \text { main pulse for } \mathrm{XR} \\
\text { or HEP generation }\end{array}$ & $<1000 \mathrm{~nm}[13],[72]$ & $<1000 \mathrm{~nm}[13],[72]$ & $\begin{array}{l}\text { not an issue for the } \\
\text { present work }\end{array}$ \\
\hline $\begin{array}{l}\tau_{\mathrm{L}} \text { main pulse for } \mathrm{XR} \\
\text { or HEP generation }\end{array}$ & $>30 \mathrm{fs}[14]$ & $\begin{array}{l}\text { for protons: > } 30 \mathrm{fs} \\
\text { FWHM [66], [72] }\end{array}$ & $\begin{array}{l}\text { not an issue for the } \\
\text { present work }\end{array}$ \\
\hline $\begin{array}{l}\text { intensity of main pulse } \\
\text { for XR or HEP } \\
\text { generation }\end{array}$ & $\begin{array}{l}10^{17}-10^{19} \mathrm{~W} / \mathrm{cm}^{2} \\
{[14],[72]}\end{array}$ & $\begin{array}{l}10^{19}-10^{20} \mathrm{~W} / \mathrm{cm}^{2} \\
{[66],[72]}\end{array}$ & $\begin{array}{l}\text { not an issue for the } \\
\text { present work }\end{array}$ \\
\hline $\begin{array}{l}\lambda_{\mathrm{L}} \text { of ripple formation } \\
\text { pulse }\end{array}$ & $<1000 \mathrm{~nm}[14],[13]$ & $<1000 \mathrm{~nm}[13],[66]$ & $\begin{array}{l}\text { present experiment: } \\
775 \mathrm{~nm}\end{array}$ \\
\hline $\begin{array}{l}\tau_{\mathrm{L}} \text { of ripple formation } \\
\text { pulse }\end{array}$ & $\begin{array}{l}>30 \mathrm{fs} \\
{[14],[13]}\end{array}$ & $\begin{array}{l}\text { for protons: > } 100 \text { fs } \\
\text { FWHM [66] }\end{array}$ & $\begin{array}{l}\text { present experiment: } \\
\sim 150 \text { fs FWHM }\end{array}$ \\
\hline $\begin{array}{l}\text { peak fluence of ripple } \\
\text { formation pulse }\end{array}$ & $\begin{array}{l}>0.2 \mathrm{~J} / \mathrm{cm}^{2} \\
{[14],[13]}\end{array}$ & $\begin{array}{l}>0.2 \mathrm{~J} / \mathrm{cm}^{2} \\
{[14],[13]}\end{array}$ & $\begin{array}{l}\text { demonstrated: } \\
0.2 \mathrm{~J} / \mathrm{cm}^{2} ; \text { applicable } \\
\text { up to } 0.8 \mathrm{~J} / \mathrm{cm}^{2}\end{array}$ \\
\hline $\begin{array}{l}\text { number of ripple } \\
\text { formation pulses } N\end{array}$ & subject of experiment & subject of experiment & $N_{o p t} \approx 90$ to 100 \\
\hline $\begin{array}{l}\lambda_{\mathrm{r}} \text { (this is an essential } \\
\text { parameter) }\end{array}$ & $\begin{array}{l}<1000 \mathrm{~nm} \\
{[14],[13]}\end{array}$ & $\begin{array}{l}<1000 \mathrm{~nm} \\
{[66],[33],[34],[72]}\end{array}$ & $600-700 \mathrm{~nm}$ \\
\hline $\begin{array}{l}h \text { (this is an essential } \\
\text { parameter) }\end{array}$ & $\begin{array}{l}>100 \mathrm{~nm} \\
{[13],[72]}\end{array}$ & $\begin{array}{l}>100 \mathrm{~nm} \\
{[13],[72],[66],[33],} \\
{[34]}\end{array}$ & $50 \mathrm{~nm}$ to $>100 \mathrm{~nm}$ \\
\hline volume of the relief & $\begin{array}{l}\text { not too much of } \\
\text { relevance }\end{array}$ & $\begin{array}{l}\text { for a foil, } h \text { should be } \\
\text { less than foil thickness } \\
{[66]}\end{array}$ & $\begin{array}{l}\text { not an issue for the } \\
\text { present work }\end{array}$ \\
\hline ripple shape & $\begin{array}{l}\text { not too much of } \\
\text { relevance }(\text { see text })\end{array}$ & $\begin{array}{l}\text { equipotential triangle is } \\
\text { a little bit better shape } \\
{[66]}\end{array}$ & $\begin{array}{l}\text { triangle with a base } \\
\text { between } 300 \text { and } \\
350 \mathrm{~nm} \text { and a height of } \\
50 \text { to } 70 \mathrm{~nm}\end{array}$ \\
\hline ripple area & $\begin{array}{l}\text { rather homog. ripple } \\
\text { region without damage }\end{array}$ & $\begin{array}{l}\text { rather homog. ripple } \\
\text { region without damage }\end{array}$ & $\begin{array}{l}\text { feasibility } \\
\text { demonstrated }\end{array}$ \\
\hline $\begin{array}{l}\text { relevant spot size for } \\
\text { the interaction process }\end{array}$ & $\begin{array}{l}\text { most important the spot } \\
\text { within FWHM }\end{array}$ & $\begin{array}{l}\text { most important the spot } \\
\text { within FWHM }\end{array}$ & $\begin{array}{l}\text { more than } 70 \% \text { of the } \\
\text { relevant area could be } \\
\text { covered by ripples } \\
\text { demonstrated }\end{array}$ \\
\hline $\begin{array}{l}\text { absorption } \\
\text { enhancement }\end{array}$ & $\begin{array}{l}u_{[66],[72]} \text { to } 100 \%(60 \% \text { in } \\
{[66]}\end{array}$ & $\begin{array}{l}\text { up to } 100 \%(60 \% \text { in } \\
{[66],[72]}\end{array}$ & $\begin{array}{l}\text { needs to be measured, } \\
\text { but not a subject of the } \\
\text { present work }\end{array}$ \\
\hline $\begin{array}{l}\text { emission enhancement } \\
\text { by factor }\end{array}$ & $\begin{array}{l}3 \text { (K- } \alpha \text { emission }) \\
{[70]}\end{array}$ & $\begin{array}{l}\text { hot el. no: factor } 5 \\
\text { fast proton no: factor } 7 \\
\text { [72] }\end{array}$ & $\begin{array}{l}\text { needs to be measured, } \\
\text { but not a subject of the } \\
\text { present work }\end{array}$ \\
\hline
\end{tabular}

Acknowledgements The authors would like to thank M. Sohn for assistance with the AFM measurements. A.A. would like to thank V. Makin for his help with the preparation of part of the reference list and for fruitful discussions. A.A. and U.T are grateful to the Deutscher Akademischer Austauschdienst (DAAD) for support by the DAAD
Gastdozentenprogramm, grant no. 57371110. We would like to thank J. Napier for critical reading of the manuscript.

Funding Open Access funding enabled and organized by Projekt DEAL. 
Open Access This article is licensed under a Creative Commons Attribution 4.0 International License, which permits use, sharing, adaptation, distribution and reproduction in any medium or format, as long as you give appropriate credit to the original author(s) and the source, provide a link to the Creative Commons licence, and indicate if changes were made. The images or other third party material in this article are included in the article's Creative Commons licence, unless indicated otherwise in a credit line to the material. If material is not included in the article's Creative Commons licence and your intended use is not permitted by statutory regulation or exceeds the permitted use, you will need to obtain permission directly from the copyright holder. To view a copy of this licence, visit http://creativecommons.org/licenses/by/4.0/.

\section{References}

1. D. Kühlke, U. Herpers, D. von der Linde, Appl. Phys. Lett. 50, 1785 (1987)

2. U. Teubner, G. Kühnle, F.P. Schäfer, Appl. Phys. Lett. 59, 2672 (1991)

3. W. Lu, M. Nicoul, U. Shymanovich, A. Tarasevitch, P. Zhou, K. Sokolowski-Tinten, D. von der Linde, M. Masek, P. Gibbon, U. Teubner, Phys. Rev. E 80, 026404 (2009)

4. A. Rousse, C. Rischel, J.C. Gauthier, Rev. Mod. Phys. 73, 17 (2001)

5. W.J. Ding, Z.M. Sheng, J. Zhang, M.Y. Yu, Phys. Plas 16, 042315 (2009)

6. Y.J. Gu, O. Klimo, P.H. Nicolai, S. Shekhanov, S. Weber, V.T. Tikhonchuk, High Power Laser Sci. Eng. 7, e39 (2019)

7. U. Teubner, W. Theobald, C. Wülker, E. Förster, Phys. Plasmas 2, 972 (1995)

8. A.A. Andreev, U. Teubner, I.V. Kurnin, E. Förster, Appl. Phys. B 70, 505 (2000)

9. K. Eidmann, J. Meyer-ter-Vehn, T. Schlegel, S. Hüller, Phys. Rev. E 62, 1202 (2000)

10. A. Grinenko, D.O. Gericke, Phys. Rev. Lett. 103, 065005 (2009)

11. H. Daido, M. Nishiuchi, A. Pirozhkov, Rep. Prog. Phys. 75, $056401(2012)$

12. A. Lübcke, A. Andreev, S. Höhm, R. Grunwald, L. Ehrentraut, M. Schnürer, Sci. Rep. 7, 44030 (2017)

13. R.V. Volkov, D.M. Golishnikov, V.M. Gordienko, A.B. Savel'ev, JETP Lett. 77, 473 (2003)

14. W.L. Kruer, The Physics of Laser Plasma Interaction (AddisonWesley, Redwood City, 1988)

15. P. Mulser, Hot Matter from High-Power Lasers (Springer, Berlin, 2020)

16. A.V. Ovchinnikov, O.F. Kostenko, O.V. Chefonov, O.N. Rosmej, N.E. Andreev, M.B. Agranat, J.L. Duan, J. Liu, V.E. Fortov, Laser Part. Beams 29, 249 (2011)

17. S. Mondal, I. Chakraborty, S. Ahmad, D. Carvalho, P. Singh, A.D. Lad, V. Narayanan, P. Ayyub, G. Ravindra Kumar, J. Zheng, Z.M. Sheng, Phys. Rev. B 83, 035408 (2011)

18. K. Sugioka, Y. Cheng (eds): Ultrafast Laser Processing, Pan Stanford Publ., Singapore 2013; R.A. Ganeev: Laser-Surface Interactions, Springer, Heidelberg

19. J. Bonse, S. Höhm, S.V. Kirner, A. Rosenfeld, J. Krüger, IEEE Sel Top in QE 23, 9000615 (2017)

20. J.E. Sipe, J.F. Young, J.S. Preston, H.M. van Driel, Phys. Rev. B 27, 1141 (1983)

21. Bonse J, Rosenfeld A, Krüger J, J. Appl. Phys. 106, 104910 (2009)

22. J. Bonse, S. Höhm, S.V. Kirner, A. Rosenfeld, J. Krüger, J. Laser Appl. 24, 042006 (2012)
23. Y. Levy, T.J.-Y. Derrien, N.M. Bulgakova, E.L. Gurevich, T. Mocek, Appl. Surf. Sci. 374, 157 (2016)

24. L.V. Zhigilei, Z. Lin, D.S. Ivanov, J. Phys. Chem. C 113, 11892 (2009)

25. D.L. Andrews, G.D. Scholes, G.P. Wiederrecht, Comprehensive Nanoscience and Nanotechnology, 4th edn. (Elsevier, Academic Press, 2011)

26. R. Buividas, M. Mikutis, S. Juodkazis, Prog. Quantum Electron. 38, 119 (2014)

27. J. Reif, O. Varlamova, F. Costache, Appl. Phys. A 92, 1019 (2008)

28. I. Gnilitskyi, V. Gruzdev, N.M. Bulgakova, T. Mocek, L. Orazi, Appl. Phys. Lett. 109, 143101 (2016)

29. Y. Jee, M.F. Becker, R.M. Walser, J. Opt. Soc. Am. B 5, 648 (1988)

30. J.-M. Romano, A. Garcia-Giron, P. Penchev, S. Dimov, Appl. Surf. Sci. 440, 162 (2018)

31. F. Fraggelakis, G. Mincuzzi, J. Lopez, I. Manek-Hönninger, R. Kling, Materials 12, 1018 (2019)

32. S. Bashir, M.S. Rafique, W. Husinsky, Nucl Instrum. Methods Phys. Res. Sect. B. 275, 1 (2012)

33. S.K. Das, A. Andreev, H. Messaoudi, J. Braenzel, M. Schnürer, R. Grunwald, J. Appl. Phys. 119, 113101 (2016)

34. A. Andreev, K. Platonov, J. Braenzel, A. Lübcke, S. Das, H. Messaoudi, R. Grunwald, C. Gray, E. McGlynn, M. Schnürer, Plasma Phys Control. Fusion 58, 014038 (2016)

35. S. Sakabe, M. Hashida, S. Tokita, S. Namba, K. Okamuro, Phys. Rev. B 79, 033409 (2009)

36. A.Y. Vorobyev, V.S. Makin, C. Guo, J. Appl. Phys. 101, 034903 (2007)

37. N. Medvedev, I. Milov, Phys. Rev. B 102, 064302 (2020)

38. I. Gnilitskyi, T. Derrien, Y. Levy, N. Bulgakova, T. Mocek, L. Orazi, Sci. Rep. 7, 8485 (2017)

39. C. Guo, Appl. Phys. Lett. 87, 251914 (2005)

40. J. Imgrunt, K. Chakanga, K. von Maydell, U. Teubner, Appl. Phys. A 123, 776 (2017)

41. S. Nolte, C. Momma, H. Jacobs, A. Tünnermann, B.N. Chichkov, B. Wellegehausen, H. Welling, J. Opt. Soc. Am. B 14, 2716 (1997)

42. J. Byskov-Nielsen, J.-M. Savolainen, M.S. Christensen, P. Balling, Appl. Phys. A 101, 97 (2010)

43. B. Neuenschwander, B. Jaeggi, M. Schmid, G. Hennig, Phys. Procedia 56, 1047 (2014)

44. C.W. Cheng, Int J Adv Manuf Technol 92, 151 (2017)

45. Y. Fuentes-Edfuf, J.A. Sánchez-Gil, M. Garcia-Pardo, R. Serna, G.D. Tsibidis, V. Giannini, J. Solis, J. Siegel, Appl. Surf. Sci. 493, 948 (2019)

46. J. Bonse, J. Krüger, J. Appl. Phys. 108, 034903 (2010)

47. M. Huang, F. Zhao, Y. Cheng, N. Xu, Z. Xu, ACS Nano 3, 4062 (2009)

48. M. Huang, Z. Xu, Laser Photon. Rev. 8, 633 (2014)

49. G.D. Tsibidis, E. Skoulas, E. Stratakis, Opt. Lett. 40, 5172 (2015)

50. www.nist.gov/pml/elemental-data-index

51. A.M. Bonch-Bruevich, M. Libenson, V. Makin, V. Trubaev, Opt. Eng. 31, 718 (1992)

52. L. Gallais, M. Rumpel, M. Moeller, T. Dietrich, T. Graf, M.A. Ahmed, Appl. Phys. B 126, 69 (2020)

53. N. Kumar, V.K. Tripathi, Phys Plasmas 14, 103108 (2007)

54. A. Macchi, Physics of Plasmas 25, 031906 (2018)

55. B. Chimier, V.T. Tikhonchuk, L. Hallo, Phys. Rev. B 75, 195124 (2007)

56. N.M. Bulgakova, R. Stoian, A. Rosenfeld, I.V. Hertel, W. Marine, E.E.B. Campbell, Appl. Phys. A 81, 345 (2005)

57. A.A. Ionin, S.I. Kudryashov, S.V. Makarov, L.V. Seleznev, D.V. Sinitsyn, Appl. Phys. A 117, 1757 (2014) 
58. E. Gamaly, A. Rode, Progress in QE 37, 5 (2013); E (Femtosecond laser-matter interactions, Pan Stanford Publ., Singapore, Gamaly, 2011)

59. A.M. Bonch-Bruevich, M.N. Libenson, Sov. J. Opt. Technol. 55, 737 (1988)

60. Y.B. Zel'dovich, Y.P. Raizer, Physics of Shock Waves and High Temperature Hydrodynamic Phenomena (Academic, New York, 1966), pp. 652-674

61. R.M. More, Adv. Atom. Mol. Phys. 21, 305 (1985)

62. L. Spitzer, Physics of Fully Lonized Gases, 2nd edn. (Interscience Wiley, New York, 1962)

63. U. Teubner, W. Theobald, C. Wülker, J. Phys. B 29, 4333 (1996)

64. H.O. Jeschke, M.E. Garcia, M. Lenzner, J. Bonse, J. Kruger, W. Kautek, Appl. Surf. Sci. 839, 197 (2002)

65. N. Rotenberg, J.E. Sipe, Phys. Rev. B 83, 045416 (2011)

66. L. Cao, Y. Gu, Z. Zhao, W. Huang, W. Zhou, X.T. He, W. Yu, M.Y. Yu, Phys. Plasmas 17, 043103 (2010)

67. A. Andreev, N. Kumar, KYu. Platonov, A. Pukhov, Phys. Plasmas 18, $103103(2011)$

68. A. Andreev, KYu. Platonov, Contrib. Plasma Phys. 53, 173 (2013)
69. D. Serebryakov, T. Volkova, E. Nerush, I. Kostyukov, Plasma Phys. Control. Fusion 61, 074007 (2019)

70. M. Blanco, M.T. Flores-Arias, C. Ruiz, M. Vranic, New J. Phys. 19, 033004 (2016)

71. A. Andreev, KYu. Platonov, Quantum Electron. 41, 515 (2011)

72. Ch. Reich, P. Gibbon, I. Uschmann, E. Förster, Phys. Rev. Lett. 84, 4846 (2000)

73. A. Andreev, K. Platonov, A. Lübke, M. Schnuerer, Phys. of Plasmas 26, 113110 (2019)

74. X. Guo (2016) Opt. Express 24: 2

75. C.L. Chang, C.W. Cheng, J.K. Chen, Appl. Surf. Sci. 469, 904 (2019)

76. M. Cerchez, M. Swantusch, M. Toncian, X.M. Zhu, R. Prasad, T. Toncian, C.H. Rodel, O. Jackel, G.G. Paulus, A.A. Andreev, O. Willi, Appl. Phys. Lett. 112, 221103 (2018)

Publisher's Note Springer Nature remains neutral with regard to jurisdictional claims in published maps and institutional affiliations. 